\title{
Sopra le coniche bitangenti alle superficie algebriche.
}

\author{
(Di Matteo Botrasso, a Torino.)
}

$\mathrm{I}$

Je questioni di pluricontatto e di contatto d'ordine superiore d'una conica con una superficie, che non sia un piano, non consta siano state risolte neppure in casi particolari.

La presente Nota apporta un primo contributo a tale classe di problemi, poichè stabilisce la condizione di bicontatto d'una conica con una superficie, $F^{n}$, generale nel suo ordine.

Il metodo che seguiremo consisterà nel ridurre, in parte, il problema nostro, relativo alle coniche dello spazio, ai problemi analoghi, risoluti dallo Zeuthen, per le coniche del piano, appoggiandoci ad un teorema dell'HALPHEN sulla teoria delle caratteristiche delle coniche. Per risolvere poi completamente la questione, ci gioveremo essenzialmente delle relazioni, che legano i caratteri d'un sistema $\infty^{1}$ di coniche col numero delle coniche del sistema che degenerano in una coppia di rette complanari, distinte o coincidenti; e quindi ci guiderà, in sostanza, il concetto stesso seguito dallo ZEUTHEN nella determinazione delle coniche d'un piano pluritangenti ad una curva di questo, dotata di sole singolarità ordinarie (*).

1. Adotteremo il calcolo coi simboli di condizione, traendo le notazioni relative dal classico trattato dallo SchUBERT $\left({ }^{* *}\right)$; epperò indicheremo

$\left(^{*}\right)$ Zeuthen, Nouvelle méthode pour déterminer les caractéristiques des systèmes cle coniques. (Nour. Ann. de Math., 2.ème série, t. V, 1866.) [亡̈ la riproduzione d'una Memoria dell'Accademia Danese, dal titolo: Nyt Bidrag til Laeren om Systemer af Keglesnit, ecc.; Copenaghen, 1865.] Queste ricerche furono poi completate dal CAYLEY, On the curves wich satisfy given conditions. (Philosophical Transactions, t. CLVIII, pag. 75, 1868; oppure: Collected, Mathematical Papers, t. VI, pag. 191.)

(**) Kalkül der abzählenden Geometrie. Leipzig, 1879.

Annali di Malematica, Serie III, tomo VIII. 
la condizione (semplice) per una conica d'aver il suo piano passante per un dato punto, o d'appoggiarsi ad una data retta, o di toccare un piano dato rispettivamente con $\mu, \nu, \rho$. Le condizioni (semplici) di degenerazione d'una conica, $o$ in una coppia di rette incidenti e distinte, $o$ in una retta doppia con due vertici ed un piano che ad essa appartengono, s'indicheranno rispettivamente con $\delta$ ed $\eta$.

Diremo inoltre $\alpha_{2}(n)$ la condizione (doppia) per una conica di toccare in due punti distinti una $F^{n}$; e $\alpha_{2}^{\prime}{ }_{2}(n)$ la condizione (pure doppia) per le coniche d'un piano di bitoccare una curva $\gamma^{n}$ di questo, generale nel suo ordine.

2. Lo ZeUthes, considerando le coniche dei sistemi elementari $\infty^{2}$ del piano, costituiti da tutte le coniche del piano che soddisfano ad una delle quattro condizioni triple $\nu^{3}, \nu^{2} \rho, \nu \rho^{2}$ e $\rho^{3}$, ha mostrato che quelle fra esse che bitoccano una $\gamma^{n}$, generale nel suo ordine, sono rispettivamente in numero di $\left({ }^{*}\right)$

$$
\begin{aligned}
\frac{1}{2} n(n-1)\left(n^{2}+3 n-6\right), & n(n-1)\left(n^{2}+3 n-8\right), \\
2 n(n-1)\left(n^{2}+n-5\right), & 2 n(n-1)\left(n^{2}-3\right) .
\end{aligned}
$$

Ora, pel teorema del Cremona sulle caratteristiche delle coniche $d^{\prime}$ un piano, la condizione per queste di bicontatto con $\gamma^{n}$, ossia il numero delle coniche d'un sistema $\infty^{2}$ del piano che bitoccano $\gamma^{n}$, dev'essere così espresso:

$$
\alpha_{2}(n)=x(n) \nu^{2}+y(n) \nu \rho+z(n) \rho^{2},
$$

ove $x, y, z$ son funzioni dell'unico carattere, $n$, di $\gamma$, e $\nu^{2}, \nu \rho, \rho^{2}$ son i caratteri del sistema $\infty^{2}$ considerato. Questi caratteri pei sistemi elementari $\infty^{2}$ del piano, prima definiti, sono eguali rispettivamente ad $(1,2,4),(2,4,4)$, $(4,4,2),(4,2,1)$, e quindi s'avranno le relazioni :

$$
\begin{aligned}
& x+2 y+4 z=\frac{1}{2} n(n-1)\left(n^{2}+3 n-6 ;\right. \\
& 2 x+4 y+4 z=n(n-1)\left(n^{2}+3 n-8\right) \\
& 4 x+4 y+2 z=2 n(n-1)\left(n^{2}+n-5\right),
\end{aligned}
$$

le quali permettono di ricavare $x, y, z$, e s'ottiene così, come espressione

(*) Zeuthes, loc. cit., pag. 200 . 
esplicita di $\alpha_{2}^{\prime}(n)$ la seguente:

$$
\alpha_{2}^{\prime}(n)=\frac{1}{4} n(n-1)\left\{2\left(n^{2}-n-1\right) \nu^{2}+(4 n-9) \nu \rho+2 \rho^{2}\right\}\left(^{*}\right) .
$$

3. Pel teorema di HaLPHEN sulle caratteristiche delle coniche dello spazio $\left({ }^{* *}\right)$ la condizione $\alpha_{2}(n)$ di bicontatto d' una conica con $F^{n}$, che esprime pure il numero delle coniche d'un sistema $\Sigma \infty^{2}$ bitangenti ad $F^{n}$, è rappresentata da un polinomio omogeneo di secondo grado in $\mu, \nu,{ }^{\rho}, \mathrm{i}$ eui coefficienti dipendono esclusivamente da $n$. Ora, se il sistema $\Sigma$ è piano, cioè se son nulli i termini che contengono la $\mu$, la $\alpha_{2}(n)$ si riduce ad $\alpha_{2}^{\prime}(n)$, e quindi si ha:

$$
\left.\begin{array}{c}
\alpha_{2}(n)=x^{\prime}(n) \mu^{2}+y^{\prime}(n) \mu \nu+z^{\prime}(n) \mu \rho+ \\
+\frac{1}{4} n(n-1)\left\{2\left(n^{2}-n-1\right) \nu^{2}+(4 n-9) \nu \rho+2 \rho^{2}\right\} .
\end{array}\right\}
$$

Per determinare i coefficienti $x^{\prime}, y^{\prime}, z^{\prime}$, che compaiono in questa formola, basterà ovviamente procurarci il valore di $\alpha_{2}(n)$ per tre convenienti sistemi $\infty^{2}$ di coniche: e ciò noi faremo considerando tutte le coniche che rispettivamente soddisfano alle condizioni sestuple $\mu^{2} \nu^{4}, \mu^{2} \nu^{2} \rho^{2}$ e $\mu \nu^{5}$; troveremo ciò̀ le coniche dello spazio soddisfacenti alle condizioni

$$
\mu^{2} \nu^{4} \alpha_{2}(n), \quad \mu^{2} \nu^{2} p^{2} \alpha_{2}(n), \quad \mu \nu^{5} \alpha_{2}(n) .
$$

A tal fine ci gioveremo delle relazioni simboliche $\left({ }^{* * *}\right)$

$$
\nu=\frac{1}{3} \delta+\frac{2}{3} \eta+\frac{4}{3} \mu, \quad \rho=\frac{2}{3} \delta+\frac{1}{3} \eta+\frac{2}{3} \mu,
$$

le quali permettono di ridurre la nostra ricerca alle questioni dei contatti di rette con $F^{n}$, e dei contatti delle coniche d'un piano con una sua curva $\gamma^{n}$.

Converrà pure tener presente la relazione $\left({ }^{* * * *}\right)$

$$
P=\mu \nu-2 \mu^{2},
$$

(*) Se si moltiplica quest'espressione di $\alpha_{2}^{\prime}(n)$ per $\rho^{3}$, s'ottiene la $4 .^{a}$ delle formole citate di Zeurhen, della quale non s'è fatto uso.

(**) HaLphen, Mémoire sur la détermination des coniques et des surfaces du second ordre. Troisième partie. (Bulletin de la Société Mathématique de France, t. II, 1874.)

(****) ScHUBERT, loc. cit., pag. 92.

$(* ; * *)$ Ibidem, pag. 95 . 
che esprime per una conica la condizione, doppia, di passaggio per un punto dato, e dalla quale segue:

$$
\mu^{2} \nu^{2}=P^{2}+4 \mu^{3} \nu
$$

4. Applicando la (4) e la prima delle (2), s'ottiene:

$$
\begin{gathered}
\mu^{2} \nu^{4} \alpha_{2}(n)=4 \mu^{3} \nu^{3} \alpha_{2}(n)+\frac{1}{3} P^{2} \nu \delta \alpha_{2}(n)+ \\
+\frac{2}{3} P^{2} \nu \eta \alpha_{2}(n)+\frac{4}{3} P^{2} \mu \nu \alpha_{2}(n),
\end{gathered}
$$

ed essendo $P^{2} \nu$ n $\alpha_{2}(n)=0, P^{2} \mu \nu \alpha_{2}(n)=\mu^{3} \nu^{3} \alpha_{2}(n)$, si potrà scrivere:

$$
\mu^{2} \nu^{4} \alpha_{2}(n)=\frac{1}{3} P^{2} \nu \delta \alpha_{2}(n)+\frac{16}{3} \mu^{3} \nu^{3} \alpha_{2}(n) \text {. }
$$

Poichè è noto del secondo membro di questa eguaglianza il secondo termine, basterà cercarne il primo, cioè $P^{2} \nu \delta^{\circ} \alpha_{2}(n)$.

A questo scopo, $\operatorname{dico} A_{1}, A_{2}$ i punti della condizione $P^{2}$, a la retta della condizione $\nu$, ed $x, y$ le due rette incidenti di cui si compone una $\delta$, soddisfacente alla condizione imposta $P^{2} \nu \alpha_{2}(n)$. I casi che si possono presentare dovranno allora, di necessità, rientrare in uno dei due seguenti: 1. $)$ una delle due rette di $\delta$, p. es. $x$, passa per i due punti $A_{1}, A_{2}$, e l'altra incontra $\left.a ; 2 .^{\circ}\right)$ una delle due rette di $\delta$, e sia la $x$, passa per l'uno dei due punti dati, p. es. $A_{1}$, e s'appoggia alla retta $a$, mentre l'altra retta, $y$, passa pel rimanente punto, $A_{2}$ : le soluzioni di questo caso, per lo scambio che si può fare dei due punti $A_{1}, A_{2}$, dovranno contarsi due volte.

Nel primo caso, perchè sia soddisfatta la condizione $\alpha_{2}(n)$, possiamo supporre o che la $y$, appoggiata ad $a$, sia bitangente ad $F^{n}$, o che le sia tangente ed inoltre il vertice di $\delta$ appartenga ad $F^{n}$. Essendo

$$
\frac{1}{2} n(n-1)(n-2)(n-3) \text { ed } \frac{1}{2} n(n-2)\left(n^{2}-9\right)
$$

l'ordine e la classe della congruenza delle rette bitangenti ad $F^{n}$, sarà $n(n+1)(n-2)(n-3)$ il numero di queste bitangenti appoggiate allo rette $a$ ed $A_{1} A_{2}$, ognuna delle quali costituisce colla $x \equiv A_{1} A_{2}$ una $\delta$ che soddisfa al problema. In secondo luogo osserviamo che per ognuno degli $n$ punti d'incontro di $x \equiv A_{1} A_{2}$ con $F^{n}$ passano precisamente $n^{2}-n-2$ rette, le quali, appoggiandosi ad $a$, toccano altrove $F^{n}$, e ciascuna di esse ci dà 
una nostra $\delta$ col vertice sulla superficie, per il qual fatto si deve considerare come una soluzione doppia (*), e così s'hanno altre $2 n\left(n^{2}-n-2\right)$ soluzioni. Il numero delle soluzioni pel primo caso è dunque $n(n-2)\left(n^{2}-1\right)$.

Nel secondo caso possiam supporre che le due rette $x, y$ o siano entrambe tangenti, o l'una di esse sia tangente ed il vertice giaccia sopra $F^{n}$, od infine che $y$ sia bitangente alla superficie. Le rette per $A_{1}$, appoggiate ad $a$, che toccano $F^{n}$ - sono $n(n-1)$, ed una qualsiasi di esse ̀̀ incontrata da $n(n-1)$ tangenti alla superficie passanti per $A_{2}$, onde nella prima ipotesi si hanno $n^{2}(n-1)^{2}$ soluzioni. Poichè poi ognuna delle dette $n(n-1)$ tangenti alla superficie per $A_{1}$ incontra ulteriormente $F^{n}$ in $n-2$ punti, sono $n(n-1)(n-2)$ le coniche $\delta$, la cui $x$ tocea $F^{n}$ ed il cui vertice giace sopra questa, le quali contano perciò come $2 n(n-1)(n-2)$ soluzioni. Il cono circoseritto da $A_{2}$ alla superficie incontra quest'ultima, fuori della curva di contatto, in una curva d'ordine $n^{2}(n-1)-2 n(n-1)$; onde sono $n(n-1)(n-2)$ le rette per $A_{2}$ appoggiate alla sezione $\gamma^{n}$ del piano $A_{1} a$ con $F^{n}$, le quali toccano altrove la superficie, ed ognuna ci dà una $\delta$, che ha la retta $y$ tangente ad $F^{n}$ ed il vertice sopra questa; epperò, in tal modo, s'ottengono $2 n(n-1)(n-2)$ soluzioni, come nella precedente ipotesi. Infine ognuna delle $\frac{1}{2} n(n-1)(n-2)(n-3)$ bitangenti ad $F^{n}$, che escon da $A_{2}$, individua una conica o che soddisfa al problema, e s'hanno cosi altrettante soluzioni. Nel secondo caso s'hanno quindi

$$
\frac{1}{2} n(n-1)\left(3 n^{2}+n--10\right)
$$

soluzioni, da contarsi, per quanto s'è detto in principio, due volte.

Sicchè concludiamo essere $P^{2} \nu \delta \alpha_{2}(n)=4 n(n-1)\left(n^{2}-3\right)$.

Sostituendo nella (5), e ricordando che è

$$
\mu^{3} \nu^{3} \alpha_{2}(n)=\frac{1}{2} n(n-1)\left(n^{2}+3 n-6\right),
$$

(*) Per quanto concerne la molteplicità colla quale una conica degenere va considerata come soddisfacente alle imposte condizioni, se queste son formate con $\vee$ e p, ei atterremo allo Schubert (loc. cit., pag. 93); e, se si tratta invece di condizioni di contatto, ei atterremo a quanto è posto rigorosamente in evidenza dallo.Zeuthen (Nouv. Ann., loc. cit.), notando che queste molteplicita non possono mutare quando si passi a considerare in luogo d'una curva piana, una superficie di cui detta curva è sezione. 
s'ottiene :

$$
\mu^{2} \nu^{4} \alpha_{2}(n)=4 n(n-1)\left(n^{2}+2 n-5\right) .
$$

5. Per la (4) e la seconda delle (2) si ha:

$$
\begin{gathered}
\mu^{2} \nu^{2} \rho^{2} \alpha_{2}(n)=4 \mu^{3} \nu \rho^{2} \alpha_{2}(n)+\frac{2}{3} P^{2} \rho j \alpha_{2}(n)+ \\
+\frac{1}{3} P^{2} \rho n \alpha_{2}(n)+\frac{2}{3} P^{2} \rho \mu \alpha_{2}(n),
\end{gathered}
$$

ed essendo identicamente

$$
P^{2} \rho \eta \alpha_{2}(n)=0, \quad P^{2} \mu \rho \alpha_{2}(n)=\mu^{3} \nu^{2} \rho \alpha_{2}(n),
$$

s'avrà la relazione:

$$
\mu^{2} \nu^{2} \rho^{2} \alpha_{2}(n)=\frac{2}{3} P P^{2} \rho \delta \alpha_{2}(n)+4 \mu^{3} \nu \rho^{2} \alpha_{2}(n)+\frac{2}{3} \mu^{3} \nu^{2} \rho \alpha_{2}(n),
$$

la quale riduce il calcolo di $\mu^{2} \nu^{2} \rho^{2} \alpha_{2}(n)$ a quello di $P^{2} \rho \delta \alpha_{2}(n)$.

Per avere questo numero dico nuovamente $A_{4}, A_{2}$ i punti della condizione $P^{2}$, ed indico con $\pi$ il piano della condizione $\rho$, sul quale deve stare il vertice di $\delta \equiv(x, y)$. Non si potranno allora presentare che $\mathrm{i}$ due casi seguenti : $\left.1 .^{\circ}\right)$ Una retta, p. es. la $x$, di $\delta$ coincide colla retta $A_{1} A_{2} ; 2 .^{\circ}$ ) una retta, $x$, di $\delta$ passa per $A_{1}$, e l'altra passa per $A_{2}$.

Nel primo caso la retta $y$ di $\delta$ dovrà passare pel punto $A_{1} A_{2} \pi \equiv x \pi$, quindi non potrà essere che una delle $\frac{1}{2} n(n-1)(n-2)(n-3)$ rette bitangenti ad $F^{n}$, uscenti da tal punto, e ci darà così altrettante soluzioni.

Nel secondo caso si dovrà supporre o che una delle due rette $x, y$ sia bitangente, ovvero che sian tangenti entrambe, oppure che l'una d'esse sia tangente (ad $F^{n}$ ), ed il vertice appartenga inoltre alla superficie. Le bitangenti ad $F^{n}$, che escono da ciascuno dei punti $A_{i}$, sono

$$
\frac{1}{2} n(n-1)(n-2)(n-3)
$$

ed ognuna individua una conica ò che soddisfa al problema, epperò s'avranro in questo modo $n(n-1)(n-2)(n-3)$ soluzioni. Le nostre coniche $\delta$, le cui due rette toccano entrambe $F^{n}$, son tante quanti sono i punti comuni alle sezioni con $\pi$ dei coni tangenti ad $F^{n}$ che escono da $A_{1}$ ed $A_{2}$, ciò̀ $n^{2}(n-1)^{2}$. S'è già notato che le rette del cono tangente ad $F^{n}$ col ver- 
tice in $A_{1}$, le quali s'appoggiano alla sezione $\gamma^{n}$ della superficie con $\pi$, ma hanno il loro punto di contatto fuori di $\pi$, sono $n(n-1)(n-2)$ : ognuna di esse ci dà una nostra $\delta$, col vertice sopra $F^{n}$, la quale va perciò contata due volte; e poichè il detto per $A_{1}$ vale anche per $A_{2}$, avremo in totale $4 n(n-1)(n-2)$ soluzioni.

Dopo ciò, osservando inoltre che per la condizione $\rho$ imposta alle $\delta$, tutte le. soluzioni ottenute devono contarsi $2^{1}$ volte, potrà concludersi essere:

$$
P^{2} \rho \text { ठ } \alpha_{2}(n)=n(n-1)\left(5 n^{2}-9 n+2\right) \text {. }
$$

Ed allora, sostituendo nella (7), tenendo pure presente che si ha.

s'ottiene :

$$
\begin{aligned}
\mu^{2} \nu^{2} \rho^{2} \alpha_{2}(n) & =n(n-1)\left(n^{2}+3 n-8\right), \\
\mu^{3} \nu \rho^{2} \alpha_{2}(n) & =2 n(n-1)\left(n^{3}+n-5\right),
\end{aligned}
$$

$$
\mu^{2} \nu^{2} \rho^{2} \alpha_{2}(n)=4 n(n-1)\left(3 n^{2}+n-11\right) .
$$

6. In virtù delle (3) e (2) si ha:

$$
\begin{gathered}
\mu \nu^{5} \alpha_{2}(n)=2 \mu^{2} \nu^{4} \alpha_{2}(n)+\frac{1}{3} P \nu^{3} \delta \alpha_{2}(n)+ \\
+\frac{2}{3} P \nu^{3} \eta \alpha_{2}(n)+\frac{4}{3} P \mu \nu^{3} \alpha_{2}(n),
\end{gathered}
$$

e, riconoscendosi essere identicamente

$$
P \nu^{3} \eta \alpha_{2}(n)=0, \quad P \mu \nu^{3} \alpha_{2}(n)=\mu^{2} \nu^{4} \alpha_{2}(n)-2 \mu^{3} \nu^{3} \alpha_{2}(n),
$$

s'avrà :

$$
\mu \nu^{5} \alpha_{2}(n)=\frac{1}{3} P \nu^{3} \delta^{2} \alpha_{2}(n)+\frac{10}{3} \mu^{2} \nu^{4} \alpha_{2}(n)-\frac{8}{3} \mu^{3} \nu^{3} \alpha_{2}(n) .
$$

Per quanto si è visto, già sappiamo essere :

$$
\begin{aligned}
& \mu^{3} \nu^{3} \alpha_{2}(n)=\frac{1}{2} n(n-1)\left(n^{2}+3 n-6\right), \\
& \mu^{2} \nu^{4} \alpha_{2}(n)=4 n(n-1)\left(n^{2}+2 n-5\right),
\end{aligned}
$$

e basterà quindi calcolare $P \nu^{3} \grave{\partial} \alpha_{2}(n)$.

A tal fine, se $A$ è il punto della condizione $P$, ed $a_{4}, a_{2}, a_{3}$ son le rette della condizione $\nu^{3}$, osserviamo che una delle rette di $\delta \equiv(x, y)$, p. es. $x$, dovrà necessariamente passare per $A$, e potrà non appoggiarsi ad alcuna delle $a_{i}$ (che dovranno allora incontrare tutte la $y$ ), od appoggiarsi ad una 
delle $a_{i}$ (mentre la $y$ s'appoggerà alle altre due), od incontrare due delle $a_{i}$ (mentre la restante $a_{i}$ sarà incidente ad $y$ ). Per $\mathrm{i}$ vari modi di scelta delle rette $a_{i}$ oguuna delle soluzioni, che s'ottengono nei due casi ultimi, andrà contata tre volte.

Nel primo caso può anzitutto supporsi che $x$ sia bitangente ad $F^{n}$, e s'hanno allora $n(n-1)(n-2)(n-3)$ soluzioni; ovvero che tanto $x$ quanto $y$ tocchino $F^{n}$, ottenendo così altre $2 n^{2}(n-1)^{2}$ soluzioni. Volendo invece che $x$ sia tangente alla superficie, ed il vertice di $\delta$ stia sopra questa, basterà osservare che tal vertice dovrà esser uno dei punti in cui la schiera delle rette appoggiate ad $a_{1}, a_{2}, a_{3}$ incontra la curva d'ordine $n(n-1)(n-2)$ d'ulteriore intersezione di $F^{n}$ col cono ad essa circoscritto da $A$, fuori della curva di contatto; epperò s'hanno in tal guisa $2 n(n-1)(n-2)$ soluzioni, da contarsi ognuna due volte. Infine, supposta la $y$ tangente ad $F^{n}$, poichè sono $2 n(n-1)$ le rette appoggiate ad $a_{1}, a_{2}, a_{3}$, che toccano la superficie, otterremo di nuovo $2 n(n-1)(n-2)$ coniche $\delta$ col vertice sopra $F^{n}$, che pure rappresentano un numero doppio di soluzioni. Onde, nel primo caso, il numero complessivo delle soluzioni è $n(n-1)\left(3 n^{2}+n-10\right)$.

Nel secondo caso, quando $x$ passando per $A$ incontra p. es. $a_{1}$, supposto che tanto $x$ quanto $y$ sian tangenti ad $F^{n}$, si hanno $2 n^{2}(n-1)^{2}$ soluziori; se invece $y$ bitocca la superficie, il numero delle soluzioni che s'ottengono è precisamente eguale a quello delle rette bitangenti ad $F^{n}$ appoggiate ad $a_{2}, a_{3}$, ciò̀ $n(n+1)(n-2)(n-3)$. - Può pure supporsi che il vertice della nostra ò appartenga ad $F^{n}$ : se questa è allora toccata dalla retta $x$, abbiamo $n(n-1)(n-2)$ di tali o da contarsi ciascuna due volte come soluzione. Vediamo ora quando $F^{n}$ è invece toccata dalla retta $y$. La rigata d'ordine $2 n(n-1)$ delle tangenti ad $F^{\prime} n$ appoggiate ad $a_{1}, a_{2}, a_{3}$ taglia la superficie, fuori della curva di contatto d'ordine $n^{2}$, in una curva d'ordine $2 n^{2}(n-2)$ : tanti son quindi i punti del piano $A a_{k}$, appartenenti ad $F^{n}$, ciascuno dei quali è vertice d'una conica $\delta$, la cui $y$ tocca la superficie; epperò in tal guisa otteniamo $4 n^{2}(n-2)$ soluzioni. - Quindi il numero delle soluzioni ottenute nel secondo caso sarà $n(n-1)\left(3 n^{2}+n-10\right)$; e, quando si scambino fra di loro le $a_{i}$, s'avranno in totale $3 n(n-1)\left(3 n^{2}+n-10\right)$ soluzioni.

Infine, nell'ultimo caso, la retta $x$, dovendo passare per $A$ ed appoggiarsi p. es. ad $a_{1}$ ed $a_{2}$, è fissa: onde la retta $y$ o sarà bitangente, oppure toccherà semplicemente la superficie ed il vertice di $\delta$ starà sopra questa. Le coniche of che soddisfano al problema sono $n(n+1)(n-2)(n-3)$ nella 
prima ipotesi, , ed $n\left(n^{2}-n-2\right)$ nella seconda; quest' ultime però si devono contare due volte. Tenendo poi conto degli scambi che si possono effettuare fra le $a_{i}$, si trae che nel terzo caso s'hanno $3 n(n-2)\left(n^{2}-1\right)$ soluzioni.

Raccogliendo s'avrà :

$$
P \nu^{3} \delta \alpha_{2}(n)=n(n-1)\left(15 n^{2}+n-4 h\right) .
$$

Quindi, sostituendo nella (9), abbiamo:

$$
\mu \nu^{5} \alpha_{2}(n)=n(n-1)\left(17 n^{2}+23 n-74\right) \text {. }
$$

7. Se moltiplichiamo ora la (1) successivamente per $\mu^{2} \nu^{4}, \mu^{2} \nu^{2} \rho^{2}, \mu \nu^{5}$, poichè è $\left({ }^{*}\right)$ :

$$
\begin{array}{llll}
\mu^{3} \nu^{5}=1 & \mu^{3} \nu^{4} \rho=2 & \mu^{3} \nu^{3} \rho^{2}=4 & \mu^{3} \nu^{2} \rho^{3}=4 \\
\mu^{2} \nu^{6}=8 & \mu^{2} \nu^{5} \rho=14 & \mu^{2} \nu^{4} \rho^{2}=24 & \mu^{2} \nu^{2} \rho^{4}=16 \\
\mu \nu^{7}=34 & \mu \nu^{6} \rho=52 & \mu \nu^{5} \rho^{2}=76 & \mu^{4} \nu^{4}=\mu^{4} \nu^{2} \rho^{2}=0
\end{array}
$$

s'ottiene :

$$
\begin{gathered}
\mu^{2} \nu^{4} \alpha_{2}(n)=y^{\prime}+2 z^{\prime}+\frac{1}{2} n(n-1)\left(8 n^{2}+20 n-47\right) \\
\mu^{2} \nu^{2} \rho^{2} \alpha_{2}(n)=4 y^{\prime}+4 z^{\prime}+2 n(n-1)\left(6 n^{2}+6 n-29\right) \\
\mu \nu^{5} \alpha_{2}(n)=x^{\prime}+8 y^{\prime}+14 z^{\prime}+n(n-1)\left(17 n^{z}+35 n-96\right) .
\end{gathered}
$$

Queste relazioni, insieme ai risultati $(6),(8),(10)$ prima ottenuti, ci dànno le equazioni :

$$
\begin{gathered}
y^{\prime}+2 z^{\prime}=-\frac{1}{2} n(n-1)(4 n-7) \\
4 y^{\prime}+4 z^{\prime}=-n(n-1)(4 n-7) \\
x^{\prime}+8 y^{\prime}+14 z^{\prime}=-2 n(n-1)(6 n-11),
\end{gathered}
$$

dalle quali, ricavando $x^{\prime}, y^{\prime}, z^{\prime}$, e sostituendo poi nella (1), si ha:

$$
\left.\begin{array}{c}
\alpha_{2}(n)=\frac{1}{4} n(n-1)\left\{8(2 n-3) \mu^{2}-2(4 n-7) \mu \nu+\right. \\
\left.+2\left(n^{2}-n-1\right) \nu^{2}+(4 n-9) \nu \rho+2 \rho^{2}\right\}\left({ }^{* *}\right) .
\end{array}\right\}
$$

$\left({ }^{*}\right)$ Schurert, loc. cit., pag. 95 .

(**) Della formula così ottenuta si può fare la verifica seguente: Se si considera il sistema, $\infty$, delle sezioni d'una data quadrica $\varphi^{2}$ coi piani d'una stella $(O)$, le coniche di Annali di Matematica, Serie III, tomo VIII. 
8. La formola a cui siamo giunti esprimerà il numero delle coniche non degeneri d'un qualsivoglia sistema $\infty^{2}$, che bitoccano una data $F^{n}$, la quale non abbia alcuna particolare relazione col sistema considerato: ossia, rimanendo fisso questo, possa supporsi che la $F^{n}$ occupi nello spazio una posizione generica. Se ciò non fosse, potrebbe accadere che il numero considerato, o diventasse infinito, o, pur restando finito, comprendesse coniche degeneri della specie $\delta, n$, o della terza specie (retta doppia con un vertice sopra di essa), con certe molteplicità. L'HaLPHen $\left(^{*}\right)$ ha dimostrato ed espresso con rigore, nel caso di sistemi semplici o quadrupli d'un piano, quand'è che, volendo escludere le coniche degeneri, è tuttavia valida la teoria delle caratteristiche; ma non essendo state estese quelle ricerche, non possiamo dire se, escludendo le coniche degeneri quando si presentano, il numero $\alpha_{2}(n)$ continui ad esser dato da una espressione del tipo di quella scritta.

9. La (11) permette di trovare tutti i numeri che si riferiscono a coniche soddisfacenti ad una o più condizioni di bicontatto con date superficie, generali nei loro ordini, e soddisfano inoltre ad altre condizioni, semplici o no, anche irriducibili $\left({ }^{* *}\right)$, delle quali si conoscono le espressioni in funzione $d i \mu, \nu, \rho$.

Come esempio, si può ricercare il numero delle coniche dello spazio che bitoccano quattro superficie $F_{1}^{n_{1}}, F_{2}^{n_{2}}, F_{3}^{n_{n_{3}}}, F_{4}^{n_{4}}$ (in posizione generica le une rispetto alle altre), cioè il numero rappresentato da $\underset{1}{\prod_{i}} \alpha_{2}\left(n_{i}\right)$.

Se si eseguisce direttamente questo prodotto, sostituendo poscia, nel risultato, alle condizioni di dimensione otto, formate con $\mu, \nu, \rho, \mathrm{i}$ loro valori

un tal sistema, che bitoccano $F^{\prime}$, non son altro che le sezioni di $\varphi^{2}$ fatte coi piani che sono bitangenti alla curva $\Gamma^{2 n}$, intersezione di $\varphi^{2}$ con $F^{n}$, cioè coi piani bitangenti al cono che da $O$ proietta la curva $\Gamma$. Ora questo cono, di classe $2 n^{2}$ con $n(n-1)$ generatrici doppie, ammette $2 n(n-1)\left(n^{2}+n-4\right)$ piani bitangenti, e tal numero s'ottiene pure dalla (11) quando in essa si pongano i caratteri del particolare sistema considerato, che si riconoscon essere:

$$
\mu^{2}=1, \quad \mu \nu=\mu \rho=2, \quad \nu^{2}=\nu \rho=\rho^{2}=4 .
$$

(*) HaLphen, Caractéristiques des systèmes de coniques. (Jour. de l'Ec. Polit.,45. ième Cahier, 1878.)

(*) Cioè, quelle condizioni che non si possono ottenere come prodotto di altre di dimensione minore, 
numerici (quali si trovano, ad es., in Schuberm, loc. cit., pag. 95), s'ottiene :

$$
\begin{aligned}
& \underset{1}{\prod_{i}^{4}} \alpha_{2}\left(n_{i}\right)=\frac{1}{4}{\stackrel{4}{n_{i}}}_{i}^{4} n_{i}\left(n_{i}-1\right) . \\
& \quad \cdot\left\{n_{1} n_{2} n_{3} n_{4}\left[23 n_{1} n_{2} n_{3} n_{4}+17 \Sigma^{4} n_{1} n_{2} n_{3}-9 \Sigma^{6} n_{1} n_{2}-47 \Sigma^{4} n_{1}+151\right]-\right. \\
& \quad-86 \Sigma^{4} n_{1}^{2} n_{2}^{2} n_{3}^{2}-42 \Sigma^{12} n_{1}^{9} n_{2}^{2} n_{3}+138 \Sigma^{12} n_{1}^{2} n_{2} n_{3}+316 \Sigma^{6} n_{1}^{2} n_{2}^{2}-156 \Sigma^{12} n_{1}^{2} n_{2}- \\
& \left.\quad-202 \Sigma^{4} n_{1} n_{2} n_{3}-576 \Sigma^{4} n_{1}^{2}-4 \Sigma^{6} n_{1} n_{2}+528 \Sigma^{4} n_{1}+416\right\},
\end{aligned}
$$

ove le sommatorie s'intendono estese a tutti i termini che si posson formare con $n_{1}, n_{2}, n_{3}, n_{4}$, del tipo di quello sçritto: l'apice posto alla sommatoria indica il numero dei termini che questa contiene.

Quando le quattro superficie $F_{i}$ abbiano tutte lo stesso ordine $n$, si ha:

$$
\begin{aligned}
& \alpha_{2}^{4}(n)=\frac{1}{4} n^{4}(n-1)^{4}\left(23 n^{8}+68 n^{7}-398 n^{6}-692 n^{5}+\right. \\
& \left.\quad+3703 n^{4}-2680 n^{3}-2328 n^{2}+2112 n+416\right) .
\end{aligned}
$$

Ponendo $n=2$, s'ottiene che le coniche dello spazio, le quali bitoccano quattro quadriche date, sono in numero di 448. 